Best Pract Res Clin Rheumatol. 2014 February ; 28(1): 143-156. doi:10.1016/j.berh.2014.01.008.

\title{
The role of arthroscopy in the management of knee osteoarthritis
}

\author{
Jeffrey N. Katz, MD, MSc, Sarah A. Brownlee, BA, and Morgan H. Jones, MD, MPH \\ Orthopedic and Arthritis Center for Outcomes Research, Department of Orthopedic Surgery (JNK \\ and SAB), Division of Rheumatology, Immunology and Allergy (JNK), Brigham and Women's \\ Hospital, Harvard Medical School; Department of Epidemiology, Harvard School of Public Health \\ (JNK); Department of Orthopedic Surgery, Cleveland Clinic (MHJ)
}

\begin{abstract}
Technological advances throughout the $20^{\text {th }}$ century enabled an increase in arthroscopic knee surgery, particularly arthroscopic debridement for OA and arthroscopic partial meniscectomy for symptomatic meniscal tear in the setting of OA. However, evaluation of the outcomes of these procedures lagged behind their rising popularity. Not until the early 2000s were rigorous outcomes studies conducted; these showed that arthroscopic debridement for OA was no better than a sham procedure in relieving knee pain or improving functional status, and that patients who underwent arthroscopic partial meniscectomy for a degenerative meniscal tear generally did not show more improvement than those who underwent sham meniscal resection or an intensive course of physical therapy. Though the number of arthroscopic knee procedures for OA performed each year has begun to decline, there remains a significant gap between the evidence and actual practice. Further investigation is needed to shore up the evidence base and bring policy and practice in line with rigorous research.
\end{abstract}

\section{Keywords}

arthroscopic surgery; arthroscopy; osteoarthritis; meniscus; meniscectomy

\section{Introduction}

This paper begins by setting the historical context for the use of knee arthroscopy in patients with osteoarthritis. We review technological developments that made arthroscopy a viable, rational therapy for patients with knee OA. We describe the early decades of knee arthroscopy use (1980s, 1990s), characterized by growing utilization and methodologically weak evaluation of treatment outcomes. We then present six important randomized

\footnotetext{
(C) 2014 Elsevier Ltd. All rights reserved.

Correspondence: Jeffrey N. Katz, MD, MSc, Orthopedic and Arthritis Center for Outcomes Research, Brigham and Women's Hospital, 75 Francis Street, Boston, MA 02115, 617-732-5338; jnkatz@ partners.org and sbrownlee@ partners.org.

Publisher's Disclaimer: This is a PDF file of an unedited manuscript that has been accepted for publication. As a service to our customers we are providing this early version of the manuscript. The manuscript will undergo copyediting, typesetting, and review of the resulting proof before it is published in its final citable form. Please note that during the production process errors may be discovered which could affect the content, and all legal disclaimers that apply to the journal pertain.
} 
controlled trials that have begun to clarify the outcomes of the two principal arthroscopic procedures done in patients with OA: arthroscopic debridement for OA per se and arthroscopic partial meniscectomy for symptomatic meniscal tear among patients with OA. We examine the extent that these trial findings have begun to influence practice and comment on barriers to more rapid translation of research findings to the clinical setting. We close by suggesting additional study questions that should be addressed by a new generation of trials.

\section{Historical Context}

\section{Development of Arthroscopic Surgery}

The precursors to modern arthroscopic surgery appeared as early as 1912, when Danish surgeon and radiologist Severin Nordentoft described endoscopic visualization of the knee joint [1]. After World War I, Japanese professor Kenji Takagi used a cystoscope to peer inside cadaver knees, and shortly thereafter a Swiss physician named Eugen Bircher reported on a series of arthroendoscopic procedures performed on the knee between 1921 and 1926 [1-3]. Also around this time, Philip Kreuscher published the first arthroscopy study in the United States and Michael Burman and colleagues reported on endoscopy in cadaveric joints $[2,4]$.

Unfortunately, this work was halted by the outbreak of the Second World War and failed to progress until Masaki Watanabe, a former student of Takagi, developed an arthroscope based on electronics and optics popularized in Japan during the war. With these technical advances, Watanabe took color photographs (1955) and performed the first arthroscopic partial meniscectomy (1962) [2]. Dr. Robert W. Jackson is largely credited with the dissemination of Watanabe's techniques in the United States, training orthopedists in North America throughout the 1970s and 1980s and publishing the first textbook of arthroscopy in 1976 [1, 2]. During this time arthroscopy benefitted from the development of more sophisticated fiber optics and television technology, which allowed the surgeon to view images on a screen rather than through direct visualization via the arthroscope. These advances ultimately freed the surgeons' hands for more complex procedures performed today such as ligament reconstruction and repair $[1,5]$.

\section{Development of MRI}

While the arthroscopic approach was certainly less invasive, costly and morbid than open arthrotomy, the explosion in use of arthroscopy awaited one further development of the late $20^{\text {th }}$ century: magnetic resonance imaging (MRI). Advanced imaging provided a noninvasive way of visualizing soft tissue structures, permitting clinicians to ascertain noninvasively whether the patient had a surgically amenable problem such as meniscal tear. MRI images confirmed clinically suspected meniscal tears or ligament injuries and provided surgeons with a surgical roadmap, facilitating the rapid uptake of knee arthroscopy in the late $20^{\text {th }}$ century.

MRI can be traced back to important discoveries in physics and chemistry by Isidor Isaac Rabi, who first described nuclear magnetic resonance (NMR) in 1938 [6]. This phenomenon - in which nuclei in a magnetic field absorb and re-emit electromagnetic radiation - allows 
for the visualization of internal body structures. In 1971 Raymond Damadian applied NMR to biomedical research and found that rat tumor tissue possessed a longer T2 relaxation time than normal tissue, which he predicted correctly could be useful in detecting malignant tumors. The first MR images of the human body were produced in 1977, and by 1983 General Electric and Siemans had produced their first full-body commercial MRI scanners [6]. With widespread access to this revolutionizing imaging technique, orthopedists could now peer inside a painful joint and search for a structural defect without taking the patient to the operating room.

\section{Introduction of and explosion in arthroscopic treatment}

The knee was the model joint for arthroscopy; both diagnostic and therapeutic applications of arthroscopic surgery debuted in the knee. By the mid-1980s, with evidence of several advantages of arthroscopic over open surgical procedures, arthroscopy became the preferred method of treatment when indicated [1,5]. Early studies showed that arthroscopy generally resulted in less pain and postoperative swelling than open procedures, and often reduced the risk of complications such as infection and arthrofibrosis [5], allowing patients to return to work and their normal activities sooner. In addition, nearly all arthroscopic procedures now can be performed in an outpatient setting, eliminating the expense of hospital stay. All of these benefits led to a rapid increase in arthroscopic surgeries performed throughout the end of the $20^{\text {th }}$ century, from 569,000 in 1994 to over 984,000 in $2006[7,8]$.

Knee arthroscopy also grew rapidly thanks in part to its close relationship with MRI, which as discussed above gave physicians unprecedented ability to localize and view structural abnormalities such as a meniscal tear inside the joint. However, the explosion in arthroscopic surgery predates evidence that has emerged in the last decade or so showing a very high prevalence of meniscal tear in both the general[9] and osteoarthritis[10] populations. This evidence highlights the frequent dissociation between the presence of a tear and patients' experience of symptoms, and suggests that surgery may be performed to resect meniscal lesions that may not be the cause of symptoms.

\section{The evidence base}

Prior to 2002, there were no rigorous, adequately powered randomized controlled trials of arthroscopic surgery vs. nonoperative therapy for knee osteoarthritis. Chang and colleagues [11] conducted an RCT to compare arthroscopic surgery and closed-needle joint lavage for patients with knee OA, but only 32 subjects were randomized. Many cohort studies were performed to evaluate arthroscopic debridement and lavage for knee OA and to evaluate arthroscopic partial meniscectomy for symptomatic meniscal tear [12-16]. However, in the absence of controls these studies were difficult to interpret.

Since 2002, six randomized controlled trials of arthroscopic management of knee OA have been published, two focusing on the efficacy of arthroscopic debridement and lavage on pain and function $[17,18]$ and four on the efficacy of arthroscopic partial meniscectomy in patients with symptomatic meniscal tear and underlying mild to moderate knee OA [19-21]. These trials are reviewed in this section and summarized in Table 1. 


\section{Trials of arthroscopic debridement and lavage for knee OA}

\section{Moseley Study}

Moseley and colleagues published a landmark trial in 2002 that fundamentally altered the role of arthroscopic surgery in patients with osteoarthritis. Noting that over a dozen uncontrolled studies had provided weak support for arthroscopic debridement and lavage, Moseley and team planned a randomized controlled trial to test the efficacy of surgery. Further recognizing that surgery may have a placebo-like effect, the authors designed the study with a sham control and strict blinding of subjects and assessors.

The study arms included arthroscopic debridement, arthroscopic lavage and a sham procedure that involved minor stabs wounds but no entry of the surgical equipment into the joint. Randomization was done in blocks defined by OA severity. To grade OA severity, the investigators rated each of the three major knee compartments from 0 to 4 (with $4=$ severe joint space narrowing) and the compartments were summed to give a summary OA severity grade ranging from $0-12$. Those patients with summary scores of 9 or greater were excluded.

Forty-four percent of eligible subjects agreed to enroll in the trial, even though they knew they had a one-in-three chance of receiving sham surgery. The authors randomized 180 subjects between 1995 and 1998 and followed the subjects for two years before unblinding them and the assessors. The authors documented early pain reduction in all three groups followed by essentially no change in pain through two years of follow-up. At no point were there clinically important differences among the three arms.

The Moseley trial has left an enormous legacy. First, it established that arthroscopic lavage and debridement were no better than sham surgery in the management of OA. Second, these investigators demonstrated that a sham trial was indeed feasible, at least in the Veterans Administration system. Finally, the study raised ethical questions about the appropriateness of sham surgery, which gave rise to a lively debate that remains unresolved [22]. Because the study did not include an arm that received no surgical intervention at all, the investigators were unable to comment on whether simply doing surgery (real or sham) was more efficacious than a non-operative placebo intervention.

\section{Kirkley study}

Kirkley and colleagues at University of Western Ontario, Canada, performed another pivotal study of the efficacy of arthroscopy for knee OA. Dr. Kirkley died tragically before the study was completed; her authors published the paper posthumously in 2008. The investigators included patients with knee osteoarthritis, excluding those with suspected or confirmed bucket handle tears and those with far advanced OA (Grade 4 changes on the Kellgren-Lawrence scale in two or more compartments). Subjects were randomized to either a standardized physical therapy regimen or the PT regimen along with arthroscopic debridement. Cartilage was debrided in $97 \%$ of subjects randomized to surgery and the meniscus was debrided in $81 \%$, testifying to the ubiquity of cartilage and meniscal flaps, tears, and debris in this population. Outcomes were assessed with the WOMAC pain and function scores. 
Remarkably, only $11 \%$ of screened subjects refused to participate. Another $21 \%$ were ineligible and $68 \%$ were randomized. While the surgical group had an initial improvement in symptoms compared to the PT group at the 3 month follow-up visit, there were no differences in improvement between the two groups at any subsequent visits. As also observed by Moseley, this trial did not identify a statistically significant or clinically meaningful difference in pain or functional status between those randomized to the PT regimen and those randomized to APM along with PT. A pre-specified analysis of subjects with complaints of locking and clicking also failed to demonstrate differences in outcome between surgical and non-operative therapy. Thus, the Kirkley study built upon the results of Moseley and colleagues and, like Moseley, also failed to demonstrate that arthroscopic debridement is superior to a typical PT regimen in patients with moderately advanced OA.

\section{Arthroscopy in meniscal tear}

The Moseley and Kirkley publications made it abundantly clear that arthroscopic lavage and debridement were no more effective than sham surgery or physical therapy in the management of symptomatic OA. However, the major indication for arthroscopy in the setting of knee OA is symptomatic meniscal tear[7]. While the Kirkley study did not exclude subjects with imaging or symptomatic evidence of meniscal tear, the focus of the study was osteoarthritis per se, not meniscal tear. Indeed, subjects were recruited on the basis of symptomatic osteoarthritis, not suspected symptomatic meniscal tear. Thus, the question of whether surgery is more effective than a standardized non-operative regimen in patients with symptomatic meniscal tear and concomitant knee OA remained unanswered even after the publication of these two landmark studies.

A number of non-randomized studies had documented the value of arthroscopic partial meniscectomy (APM) in patients with meniscal tear [23-25]; however, as with the early literature on surgery for OA, these studies were limited by uncontrolled observations of heterogeneous interventions performed on generally small samples. Further, and perhaps most important, much of the literature on surgery for meniscal tears had been conducted in younger persons with otherwise normal knees. Yet, increasingly APM was being performed on middle aged and older individuals with concomitant knee OA. It is unclear whether the presentation, natural history and optimal treatment for these degenerative tears differ from those associated with traumatic tear in persons in their 20s and 30s.

Four studies have been published in the last several years that begin to address these questions about the role of surgery in persons with meniscal tear and concomitant OA.

\section{Herrlin Study}

Herrlin and colleagues in Stockholm, Sweden enrolled 96 subjects 45-64 years of age with knee pain, meniscal tear on MRI and radiographic OA with, at most, minor joint space narrowing. Subjects were randomized to a rigorous exercise regimen alone or to the same exercise regimen with arthroscopic partial meniscectomy. The exercise regimen was supervised by a physical therapist twice a week for two months. Outcomes were assessed at 2, 6, 24 and 60 months with the KOOS (Knee Injury and Osteoarthritis Outcome Score) measures of pain, ADL, recreational and sports activities and knee related quality of life. 
In intention to treat analyses, the two randomized groups both improved considerably over the first six months and maintained improvements in pain and functional status over 60 months. The findings did not reveal statistically significant or clinically relevant differences between the two randomized groups at any time point. Of note, however, about $30 \%$ of the subjects randomized to the non-operative arm had persistent pain and crossed over to have surgery. These subjects who crossed over to have surgery had similar outcomes to those randomized to receive surgery at the outset. The investigators suggested that their findings supported initial treatment with non-operative therapy in middle-aged individuals with symptomatic meniscal tear and concomitant osteoarthritis, with subsequent surgery in those who failed to improve.

\section{MeTeOR}

The MeTeOR (Meniscal Tear in Osteoarthritis Research) Trial shared many design similarities to the study of Herrlin et al. MeTeOR enrolled patients age 45 or greater with meniscal tear documented on MRI and underlying osteoarthritic change documented on radiograph or MRI. Patients with Kellgren-Lawrence Grade 4 OA (more than 50\% joint space narrowing) were excluded. Subjects were randomized to receive either a standardized PT regimen that emphasized strengthening or arthroscopic partial meniscectomy with postoperative PT. The primary outcome was change in WOMAC Function Scale between baseline and six months. Secondary outcomes included the KOOS Pain Scale and these same outcomes at 12 months.

The MeTeOR Trial was considerably larger than the trial of Herrlin et al, with 351 subjects randomized in seven centers. The results documented that subjects randomized to APM and those randomized to PT both improved considerably in the first six months with no statistically significant or clinically important differences between randomized groups at six or twelve months of follow up. As in Herrlin et al, however, about $30 \%$ of MeTeOR subjects crossed over from non-operative therapy to surgery.

\section{Yim Study}

In 2013, Yim and colleagues published a randomized controlled trial of arthroscopic partial meniscectomy vs. a non-operative regimen focused on strengthening in 102 subjects with symptomatic meniscal tear. Patients were followed for 24 months with a similar set of outcome assessments as in the Herrlin and MeTeOR trials. Yim and colleagues also found that in the intention to treat analysis, both groups improved considerably with no clinically important or statistically significant differences in improvement in any of the key outcome measures. In contrast to the other two studies, however, only one subject in the study of Yim and colleagues crossed over from the non-operative arm to receive surgery. In addition, it is noteworthy that over $60 \%$ of eligible patients agreed to participate in Yim's study, compared to $<30 \%$ in MeTeOR. The reason(s) for these differences remain unclear and warrant further investigation.

\section{Sihvonen Study}

In late 2013 Sihvonen and colleagues published a randomized controlled trial of arthroscopic partial meniscectomy vs. sham surgery for degenerative meniscal tear for 146 
patients aged 35 to 65 years with symptoms of degenerative medial meniscal tear and no knee osteoarthritis. Subjects were assigned to either arthroscopic partial meniscectomy or arthroscopic surgery with sham meniscal resection. Like those of Moseley and Kirkley, Sihvonen's subjects in both arms showed substantial improvement in pain and function in the first two months following surgery with no significant or clinically important differences between arms in the change from baseline to 12 months in any primary outcome.

\section{Does the evidence influence practice?}

Ultimately, effectiveness research should reduce the gap between what we know and what we do. A limited number of investigations have examined whether the Moseley and Kirkley studies, which showed no advantage of surgery in treatment of osteoarthritis, have influenced the utilization of these procedures. Potts and colleagues addressed this question using the American Board of Orthopedic Surgery database, which includes six month logs submitted by each surgeon taking the Board examination.[26] This database provides insight into practice patterns among predominantly younger orthopedic surgeons. Data are available from 1999. Potts and colleagues used the database to determine whether the reported use of arthroscopic knee surgery for osteoarthritis declined following the publication of the Moseley trial in 2002.

Indeed, the authors noted that the frequency of arthroscopic surgery for OA peaked in 2001 and declined from that point through 2009, the last year of data used in this study. The number of cases of arthroscopy for OA per surgeon declined by $40 \%$ over that period, while the proportion of arthroscopy cases in which chondroplasty was performed also declined by $40 \%$ in the same period. The proportion of all surgery accounted for by knee arthroscopy declined by $33 \%$. These findings provide intriguing, suggestive evidence that the Moseley results may indeed have influenced practice, or at least the reported activities of surgeons sitting for the ABOS Boards.

Kim and colleagues [7] examined the US National Ambulatory Surgical Database for changes in utilization of ambulatory orthopedic surgical procedures from 1996 to 2006. (These are the only two instances in the last 17 years that this survey has been conducted). While the number of knee arthroscopies increased $49 \%$ over the ten year period, the number performed for OA declined 18\%, from 69,092 to 56,392 (See Figure 1). The proportion of all knee arthroscopies done for osteoarthritis declined from $10 \%$ to $6 \%$. In contrast, the number of all arthroscopies done for meniscal tears increased 25\%, from 371,561 to 465,914 . These data demonstrate that even though knee arthroscopies were on the rise during this ten year period, they were done less frequently for OA, again suggesting that the Moseley trial (though not the APM trials discussed above, which post-date Kim's analysis) may have had a role in catalyzing change in practice. The Medicare program, which insures virtually all US citizens age 65 and greater, stopped reimbursing physicians for arthroscopies done for knee OA in 2004. This policy change likely had a major role in curbing utilization. However, it is unclear whether surgeons simply used different codes (such as meniscal tear) when performing arthroscopy for OA, or actually performed fewer of these procedures in the OA population. 
Holmes and colleagues performed [27] a similar analysis to that of Kim at el, using the Florida Ambulatory State Database from 2000 to 2008. These authors also found the rate of arthroscopic surgery for OA declined 39\% from 2000 to 2008. This reduction was evident among both privately and publicly insured patients. Howard and colleagues [28] also used the Florida State Ambulatory Surgery Database to analyze trends in knee arthroscopy rates from 1998 -2010. They were able to assess the impact of the publication of the Moseley trial in 2002, the release of the CMS decision memo denying coverage for arthroscopic debridement and lavage in 2003, and the publication of the Kirkley trial in 2008. They noted a decline in arthroscopic debridement following each of these events, while overall rates of other knee arthroscopies were actually increasing. Bohensky and colleagues [29] performed a similar analysis in Victoria, Australia, examining administrative data from 2000 to 2009. In contrast to the US findings noted above, these authors did not observe a reduction in use of knee arthroscopy during this period, albeit there was no change in reimbursement policy for arthroscopy as occurred in the US in 2004. Harris et al [30], from New South Wales, Australia, observed that the rate of TKA occurring in the two years following knee arthroscopy declined from 2000-2008, suggesting fewer arthroscopies were being performed on those with advanced OA.

\section{Influence on Practice Guidelines}

Several organizations have published guidelines for management of osteoarthritis, including the American College of Rheumatology (ACR), American Academy of Orthopedic Surgeons (AAOS), Osteoarthritis Research Society International (OARSI) and the National Institute for Health and Clinical Excellence (NICE) of the National Health Service, United Kingdom [31-35]. Each of these organizations has published osteoarthritis treatment guidelines in the last several years. Table 2 lists the components of these guidelines pertinent to the use of arthroscopic surgery for osteoarthritis. As the Table indicates, each set of guidelines specifically recommends against the use of arthroscopic surgery for the management of osteoarthritis, unless there is evidence of meniscal tear as the cause of symptoms. The lone exception is the American College of Rheumatology 2012 Guidelines, which do not comment on use of surgery.

In summary, it appears that the results of the Moseley trial (and perhaps the Kirkley trial as well) influenced practice. Following the publication of the Moseley trial in 2002 the number of arthroscopies performed for knee OA appeared to decline as reflected in both the number of arthroscopies performed for OA reported to the American Board of Orthopedic Surgeons and as well as utilization trends in the National Ambulatory Surgical Database. Furthermore, OA practice guidelines developed by professional organizations and payers have discouraged use of arthroscopic debridement and lavage for knee OA.

It is likely too early to determine if the studies of arthroscopic partial meniscectomy have influenced practice. Herrlin et al published their 6 and 24 month results in 2007, and their five year results in 2013. The MeTeOR Trial and that of Yim and colleagues and of Sihvonen and colleagues, were published in 2013. Before we can discern the effects of these trials on practice, time will have to pass for the trials to disseminate through the professional community and for the accumulation of sufficient data on utilization of these procedures. 


\section{Barriers to greater impact of evidence upon practice}

There has been considerable scholarly examination of the gap between clinical evidence and clinical practice. Haynes and Haines [36] addressed this issue in 1999 and their comments remain relevant: These authors noted that once research evidence is generated, it must be 1) synthesized into the literature, 2) developed into evidence based clinical policies; and 3) applied by clinicians in practice. Barriers may arise at each step and the progression from one step to the next takes time. Grol and Grinshaw [37] wrote in 2003 on difficulties that arise in translating evidence and guidelines to practice. These authors highlighted a range of potential barriers including financial disincentives for providers to change practice style, difficulty overcoming the status quo, disagreement among key opinion leaders, information overload among providers and a range of other factors. We comment further on strategies for overcoming such barriers in the Future Directions section below.

These conceptual pieces provide a foundation for assessing the progress to implementation, barriers and potential remedies in the case of arthroscopy for OA and for meniscal tear in the OA population. Regarding management of OA per se, the critical evidence establishing that arthroscopy is not efficacious was generated in the early (Moseley) and late (Kirkley) 2000's. Recent literature syntheses on OA treatment have included the Moseley and Kirkley trials and these trial findings have indeed been integrated into practice guidelines by major professional organizations, as noted in this paper [31-35]. For these guidelines to be applied in practice, the clinicians need to know of them and to be able to access them readily in their practice environments. Haynes speculated 15 years ago that increasing use of more facile electronic systems in practice would help bridge the gap between practice guidelines and their intended audience [36]. This process is underway, as many electronic medical systems have decision support tools embedded that provide point of service access to guidelines. Indeed, the national, state and ABOS data cited earlier suggest that clinicians are beginning to act upon the guidelines, which uniformly recommend against arthroscopic debridement for OA.

The literature on APM for symptomatic meniscal tear is less mature. Three of the four trials were published in 2013. To date, reviews and literature syntheses on management of meniscal tear in middle aged adults have not included these 2013 studies, though this will no doubt change. As the studies become more established in the literature their findings will become integrated into treatment guidelines. It is difficult to predict whether authoritative guidelines will recommend against APM based on the evidence that it appears to offer not advantages over sham or over a rigorous PT program -- or whether guidelines will portray the choice as a shared decision for patients to make with their physicians, given the improvement seen in both the APM groups and the comparators in each of the trials. This issue may play out differently across countries, depending on the propensity within each culture to emphasize patient and physician choice or efficient use of resources. This point reinforces the need for formal economic analyses to guide policy.

As noted by Haynes and others, the translation from evidence to practice will never be complete, as treatment decisions ultimately involve real patients with nuanced symptoms, signs, preferences, social supports and other factors that influence treatment decisions. There 
may be circumstances in which physicians make decisions that conflict with recommendations because particular patient factors are compelling.

\section{Future Directions}

The trials reviewed in this paper have helped to clarify several of the key questions that characterized the field in the 1990s. The studies of Moseley et al and Kirkley et al argue compellingly that arthroscopic lavage and debridement should not be done for knee OA per se. The work of Potts et al and Kim et al suggest that indeed fewer such procedures have been done for OA since the publication of the Moseley trial. Interpretation of the arthroscopic partial meniscectomy trials is less straightforward and their effect on the field less clear at this point. The studies suggest treating with a standardized, rigorous PT regimen yields outcomes similar to treatment with arthroscopic partial meniscectomy. We do not know, however, whether the rigorous PT regimens used in these trials are superior to placebo or to a much less intensive approach to PT. We also do not know which patients are going to improve with PT and which are going to have continued symptoms and require APM. As PT is costly, additional studies to clarify these issues would be useful. Further, it is tempting to conclude from these studies that the efficacy of APM among those who initially fail PT will be similar to the efficacy among those randomized to APM. However this too is unknown. The efficacy of APM vs. continued, intensified non-operative therapy among those who fail to respond to initial treatment with PT merits further study.

Finally, with four distinct, rigorous trials now available, attention should turn to disseminating findings and overcoming barriers to bridging evidence and practice. The articulation of guidelines for use of APM is a critical first step. As noted, we envision that such guidelines will urge restraint in use of APM. Whereas financial incentives may pose a barrier to implementing recommendations to reduce use of APM, the policy context is changing quickly. As health systems in the US and elsewhere adopt an accountable care framework, in which organizations receive fixed payments to take care of populations, physicians may increasingly face incentives to perform fewer and not more resource intensive treatments.

\section{Acknowledgments}

Grant support: NIH P60AR04478; R01AR05557

\section{References}

1. Bigony L. Arthroscopic surgery: a historical perspective. Orthop Nurs. 2008; 27(6):349-54. quiz 355-6. [PubMed: 19057361]

2. Jackson RW. Presidential Guest Speaker's Address: Quo Venis Quo Vadis: The Evolution of Arthroscopy. Arthroscopy: The Journal of Arthroscopic \& Related Surgery. 1999; 15(6):680-685.

3. Kieser CW, Jackson RW. Eugen Bircher (1882-1956) the first knee surgeon to use diagnostic arthroscopy. Arthroscopy. 2003; 19(7):771-6. [PubMed: 12966386]

4. Burman MS. Arthroscopy or the direct visualization of joints: an experimental cadaver study. 1931. Clin Orthop Relat Res. 2001; (390):5-9. [PubMed: 11550876]

5. Treuting R. Minimally invasive orthopedic surgery: arthroscopy. Ochsner J. 2000; 2(3):158-63. [PubMed: 21765685] 
6. Ai T, et al. A historical overview of magnetic resonance imaging, focusing on technological innovations. Invest Radiol. 2012; 47(12):725-41. [PubMed: 23070095]

7. Kim S, et al. Increase in Outpatient Knee Arthroscopy in the United States: A Comparison of National Surveys of Ambulatory Surgery, 1996 and 2006. The Journal of Bone \& Joint Surgery. 2011; 93(11):994-1000. [PubMed: 21531866]

8. Kozak LJ, et al. Ambulatory surgery in the United States, 1994. Adv Data. 1997; (283):1-15. [PubMed: 10172944]

9. Englund M, et al. Incidental Meniscal Findings on Knee MRI in Middle-Aged and Elderly Persons. New England Journal of Medicine. 2008; 359(11):1108-1115. [PubMed: 18784100]

10. Bhattacharyya T, et al. The Clinical Importance of Meniscal Tears Demonstrated by Magnetic Resonance Imaging in Osteoarthritis of the Knee*. The Journal of Bone \& Joint Surgery. 2003; 85(1):4-9. [PubMed: 12533565]

11. Chang RW, et al. A randomized, controlled trial of arthroscopic surgery versus closed-needle joint lavage for patients with osteoarthritis of the knee. Arthritis Rheum. 1993; 36(3):289-96. [PubMed: 8452573]

12. Sprague NF 3rd. Arthroscopic debridement for degenerative knee joint disease. Clin Orthop Relat Res. 1981; (160):118-23. [PubMed: 7285411]

13. Baumgaertner MR, et al. Arthroscopic debridement of the arthritic knee. Clin Orthop Relat Res. 1990; (253):197-202. [PubMed: 2317974]

14. Gross DE, et al. Arthroscopic treatment of degenerative joint disease of the knee. Orthopedics. 1991; 14(12):1317-21. [PubMed: 1784548]

15. McLaren AC, et al. Arthroscopic debridement of the knee for osteoarthrosis. Can J Surg. 1991; 34(6):595-8. [PubMed: 1747839]

16. Rand JA. Role of arthroscopy in osteoarthritis of the knee. Arthroscopy. 1991; 7(4):358-63. [PubMed: 1755883]

17. Moseley JB, et al. A Controlled Trial of Arthroscopic Surgery for Osteoarthritis of the Knee. New England Journal of Medicine. 2002; 347(2):81-88. [PubMed: 12110735]

18. Kirkley A, et al. A Randomized Trial of Arthroscopic Surgery for Osteoarthritis of the Knee. New England Journal of Medicine. 2008; 359(11):1097-1107. [PubMed: 18784099]

19. Herrlin S, et al. Arthroscopic or conservative treatment of degenerative medial meniscal tears: a prospective randomised trial. Knee Surgery, Sports Traumatology, Arthroscopy. 2007; 15(4):393401.

20. Katz JN, et al. Surgery versus Physical Therapy for a Meniscal Tear and Osteoarthritis. New England Journal of Medicine. 2013; 368(18):1675-1684. [PubMed: 23506518]

21. Yim JH, et al. A Comparative Study of Meniscectomy and Nonoperative Treatment for Degenerative Horizontal Tears of the Medial Meniscus. The American Journal of Sports Medicine. 2013; 41(7):1565-1570. [PubMed: 23703915]

22. Horng S, Miller FG. Is Placebo Surgery Unethical? New England Journal of Medicine. 2002; 347(2):137-139. [PubMed: 12110744]

23. Katz JN, et al. Predictors of functional outcomes after arthroscopic partial meniscectomy. J Rheumatol. 1992; 19(12):1938-42. [PubMed: 1294743]

24. Katz JN, et al. The MeTeOR Trial (Meniscal Tear in Osteoarthritis Research): Rationale and design features. Contemporary Clinical Trials. 2012; 33(6):1189-1196. [PubMed: 22968127]

25. Meredith DS, et al. Factors predicting functional and radiographic outcomes after arthroscopic partial meniscectomy: A review of the literature. Arthroscopy: The Journal of Arthroscopic \& Related Surgery. 2005; 21(2):211-223.

26. Potts A, et al. Practice Patterns for Arthroscopy of Osteoarthritis of the Knee in the United States. The American Journal of Sports Medicine. 2012; 40(6):1247-1251. [PubMed: 22562787]

27. Holmes R, et al. Effect of Evidence and Changes in Reimbursement on the Rate of Arthroscopy for Osteoarthritis. The American Journal of Sports Medicine. 2013; 41(5):1039-1043. [PubMed: 23460330] 
28. Howard D, Brophy R, Howell S. Evidence of no benefit from knee surgery for osteoarthritis led to coverage changes and is linked to decline in procedures. Health Aff (Millwood). 2012; 31(10): 2242-9. [PubMed: 23048105]

29. Bohensky MA, et al. Trends in elective knee arthroscopies in a population-based cohort, 20002009. Med J Aust. 2012; 197(7):399-403. [PubMed: 23025737]

30. Harris IA, et al. Trends in knee arthroscopy and subsequent arthroplasty in an Australian population: a retrospective cohort study. BMC Musculoskelet Disord. 2013; 14:143. [PubMed: 23617303]

31. Excellence, N.I.o.H.a.C. Osteoarthritis: The care and management of adults with osteoarthritis. 2008.

32. Zhang W, et al. OARSI recommendations for the management of hip and knee osteoarthritis, Part II: OARSI evidence-based, expert consensus guidelines. Osteoarthritis and Cartilage. 2008; 16(2): 137-162. [PubMed: 18279766]

33. Zhang W, et al. OARSI recommendations for the management of hip and knee osteoarthritis: Part III: changes in evidence following systematic cumulative update of research published through January 2009. Osteoarthritis and Cartilage. 2010; 18(4):476-499. [PubMed: 20170770]

34. Brown GA. AAOS Clinical Practice Guideline: Treatment of Osteoarthritis of the Knee: EvidenceBased Guideline, 2nd Edition. Journal of the American Academy of Orthopaedic Surgeons. 2013; 21(9):577-579. [PubMed: 23996989]

35. Hochberg MC, et al. American College of Rheumatology 2012 recommendations for the use of nonpharmacologic and pharmacologic therapies in osteoarthritis of the hand, hip, and knee. Arthritis Care \& Research. 2012; 64(4):465-474. [PubMed: 22563589]

36. Haynes B, Haines A. Barriers and bridges to evidence based clinical practice. BMJ. 1998; 317(7153):273-276. [PubMed: 9677226]

37. Grol R, Grimshaw J. From best evidence to best practice: effective implementation of change in patients' care. The Lancet. 2003; 362(9391):1225-1230.

38. Herrlin SV, et al. Is arthroscopic surgery beneficial in treating non-traumatic, degenerative medial meniscal tears? A five year follow-up. Knee Surg Sports Traumatol Arthrosc. 2013; 21(2):358-64. [PubMed: 22437659]

39. Sihvonen R, et al. Arthroscopic Partial Meniscectomy versus Sham Surgery for a Degenerative Meniscal Tear. New England Journal of Medicine. 2013; 369(26):2515-2524. [PubMed: 24369076] 


\section{Practice Points}

- For now, it is reasonable to explain to patients with knee OA that arthroscopy should not play a role in their care unless they have clinical and imaging evidence of symptomatic meniscal tear; that is, a tear causing mechanical symptoms, e.g. catching, locking, popping and the like.

- In patients with OA and symptomatic meniscal tear, a course of physical therapy focused on strengthening is a reasonable recommendation, as the majority of patients will experience improvement from PT alone and there is no compelling evidence that APM is superior to a rigorous PT program, or to sham APM for that matter.

- Patients who fail to improve following a course of PT can be referred for APM, though no level-one evidence exists that APM is more useful than continued PT and observation in these patients. 


\section{Research Agenda}

- Given the comparable outcomes of APM and intensified PT for the treatment of meniscal tear in the context of OA, as well as the considerable cost of an intensive PT regimen, less rigorous PT should be evaluated for its ability to reduce pain and improve function as well as more intense PT at a lower cost.

- Whether APM is more effective than continued PT and observation in patients who do not respond to initial PT is uncertain and should be investigated.

- Continued research is needed to document whether the APM trials published in the last year reduce utilization of this procedure, as might be predicted based on the negative results of each of these trials. 


\section{Summary}

The popularity of arthroscopic knee surgery rose quickly during the second half of the $20^{\text {th }}$ century thanks to technological advances, including the development of MRI. However, the increase in number of procedures performed, especially arthroscopic debridement for OA and arthroscopic partial meniscectomy for degenerative meniscal tear, outstripped rigorous tracking of procedure outcomes. By the end of the 1990s the need for randomized controlled trials that assessed the efficacy of these treatments was recognized. Trials thus far have suggested arthroscopy for OA and APM for degenerative meniscal tear and no more effective than a sham procedure or intensive physical therapy. In the wake of these results, more investigation is needed to ascertain the most efficacious and cost-effective ways to treat these conditions. 


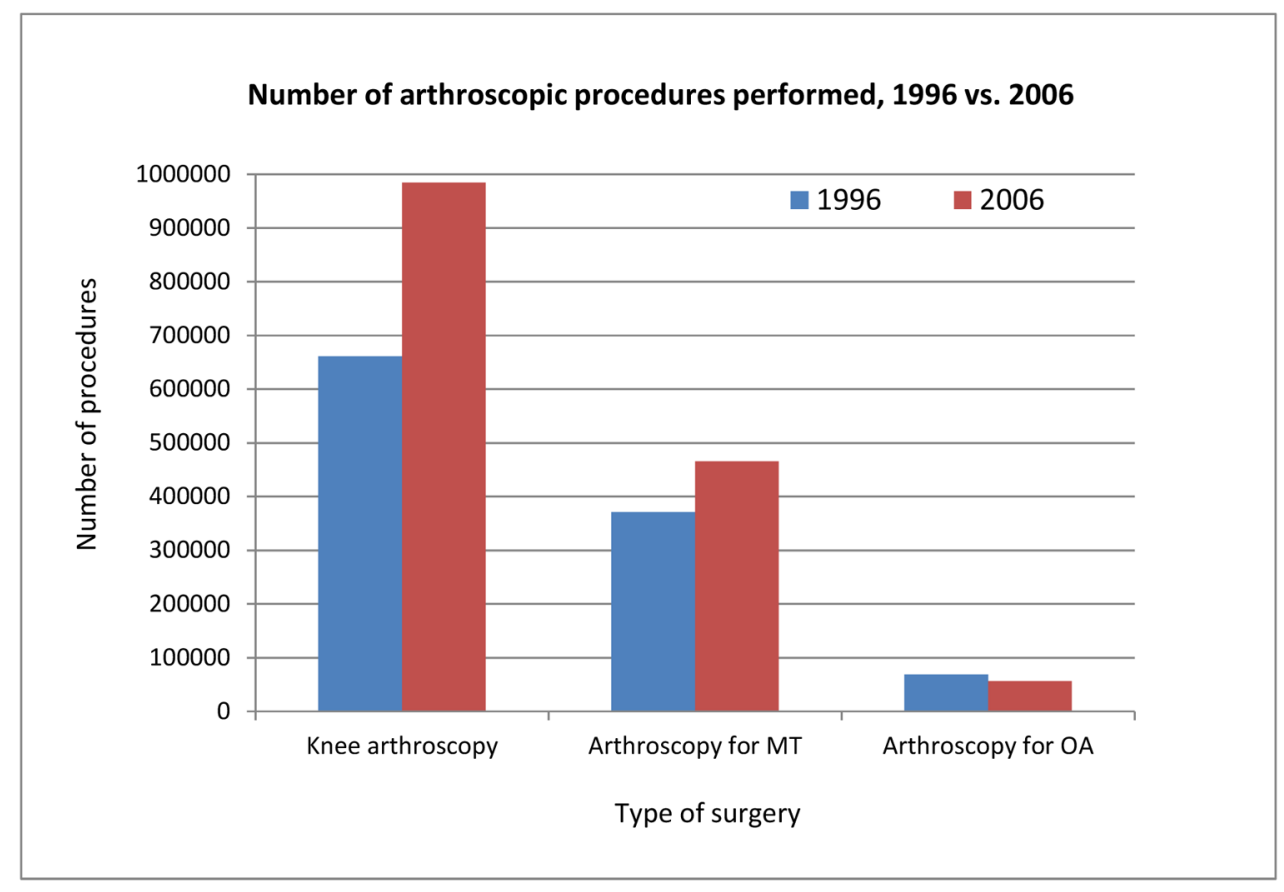

Figure 1.

Frequency of knee arthroscopy (overall) and of arthroscopy for meniscal tear and for osteoarthritis in 1996 and in 2006* *Data from Kim, et al.[7] 


\begin{tabular}{|c|c|c|c|c|}
\hline & 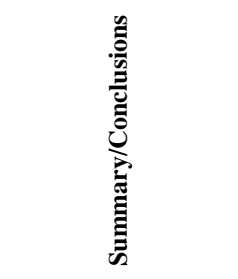 & 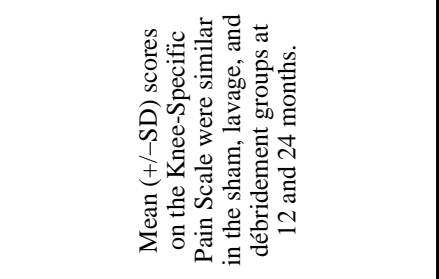 & 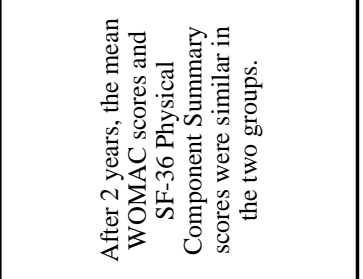 & 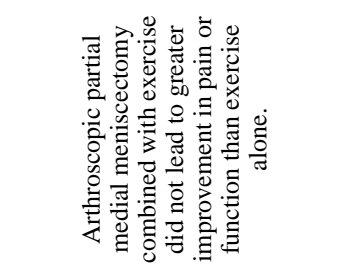 \\
\hline & 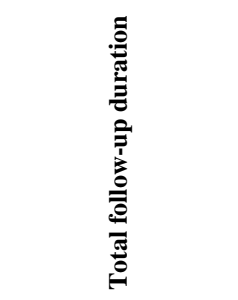 & 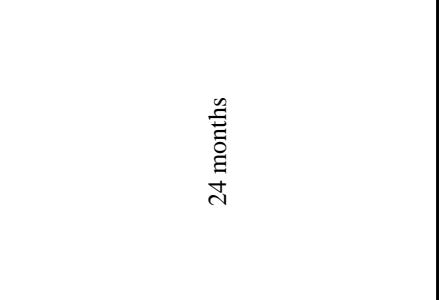 & 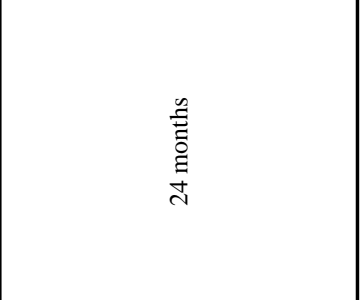 & $\begin{array}{l}\stackrel{\tilde{\sharp}}{D} \\
\stackrel{2}{n}\end{array}$ \\
\hline & 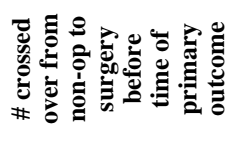 & 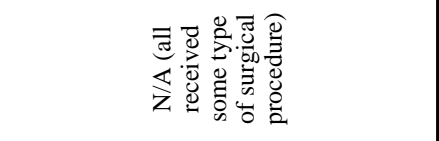 & 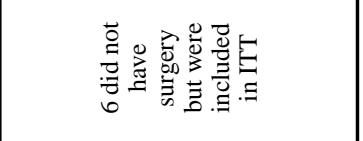 & 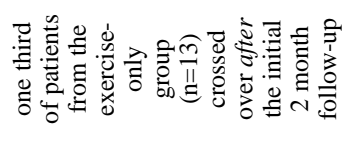 \\
\hline$\cong$ & 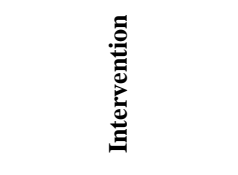 & 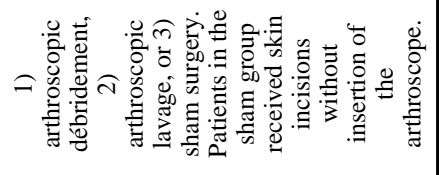 & 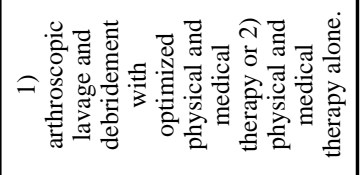 & 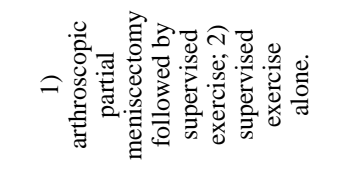 \\
\hline 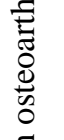 & 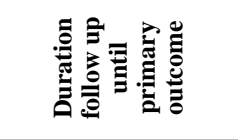 & 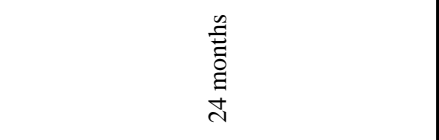 & 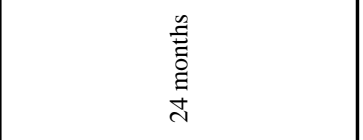 & 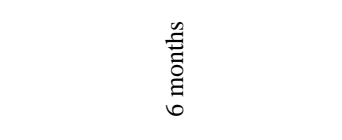 \\
\hline 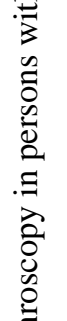 & 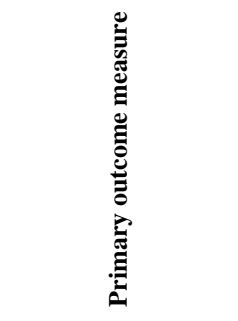 & 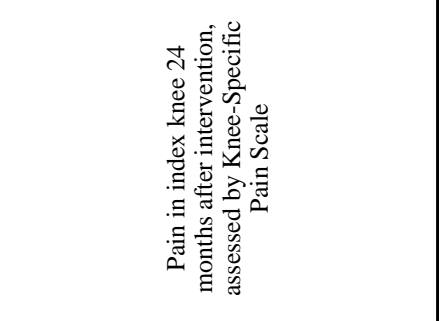 & 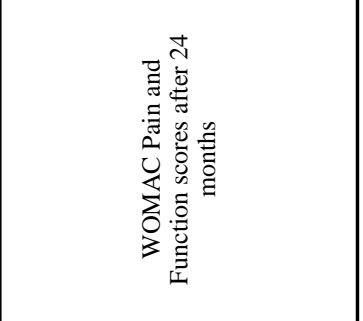 & 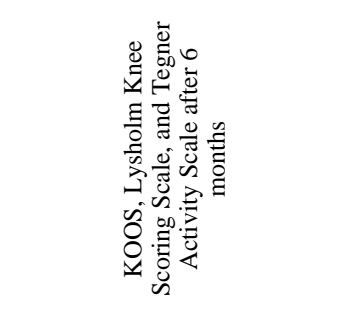 \\
\hline 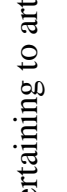 & 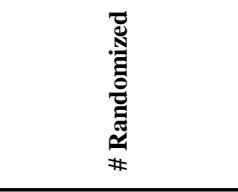 & $\stackrel{\infty}{\sim}$ & $\stackrel{\infty}{\stackrel{\infty}{\infty}}$ & gे \\
\hline 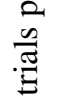 & 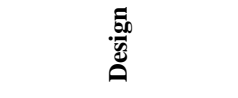 & $\underset{\approx}{E}$ & $\underset{\varkappa}{E}$ & $\underset{\approx}{E}$ \\
\hline 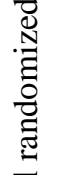 & $\stackrel{\cong}{\Xi}$ & 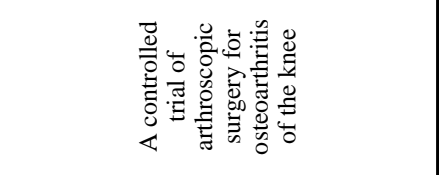 & 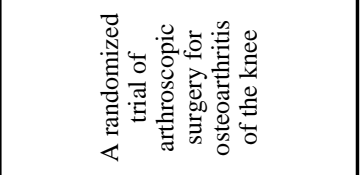 & 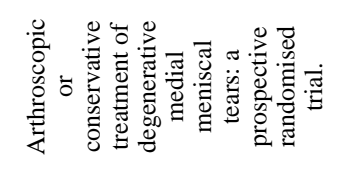 \\
\hline 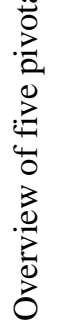 & 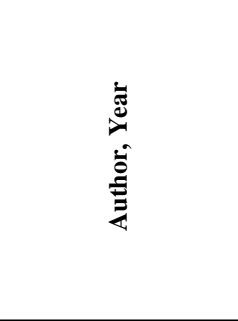 & 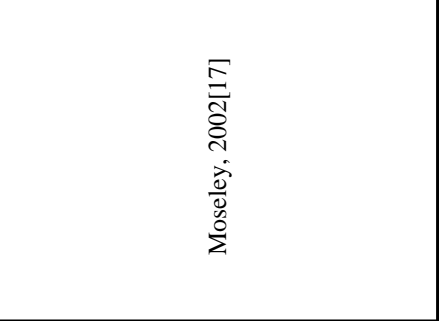 & 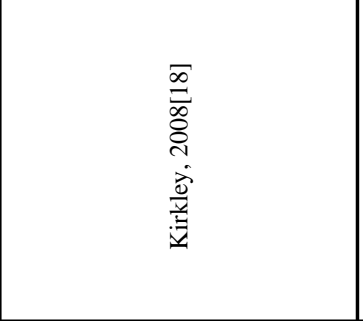 & 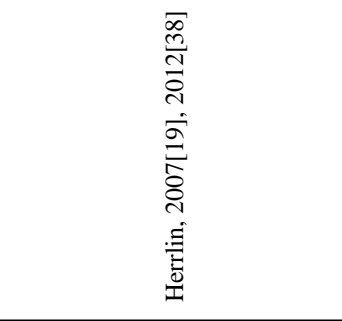 \\
\hline
\end{tabular}

Best Pract Res Clin Rheumatol. Author manuscript; available in PMC 2015 February 01. 


\begin{tabular}{|c|c|c|c|}
\hline Katetet al. & 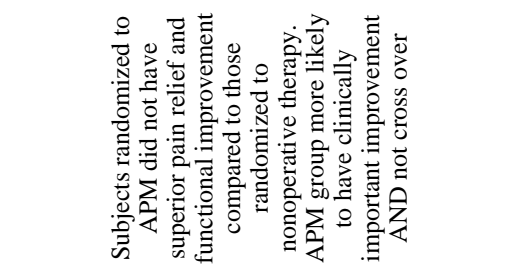 & 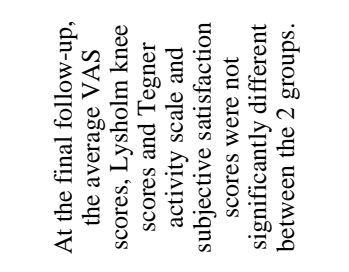 & 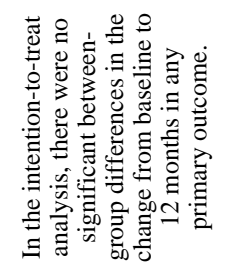 \\
\hline 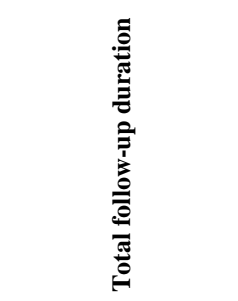 & 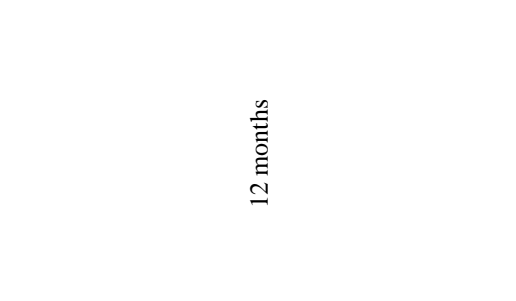 & 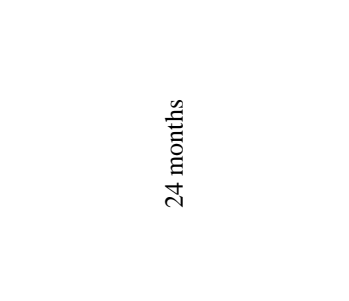 & 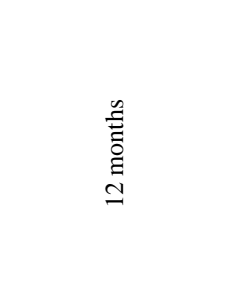 \\
\hline 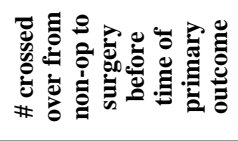 & $\begin{array}{l}\text { बे } \\
\text { कृ } \\
\bar{n}\end{array}$ & - & $\mathbb{z}$ \\
\hline 咅 & 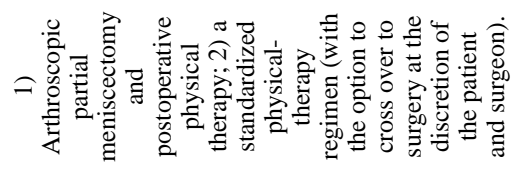 & 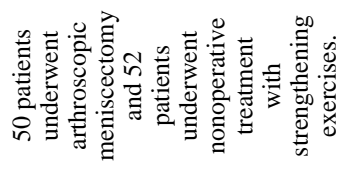 & 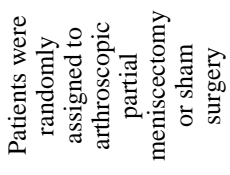 \\
\hline 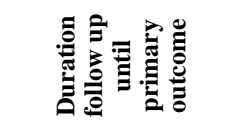 & $\begin{array}{l}\text { 气 } \\
\text { 音 } \\
\text { हू }\end{array}$ & $\begin{array}{l}\text { 吉 } \\
\text { 音 } \\
\text { d }\end{array}$ & 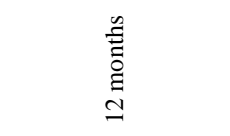 \\
\hline 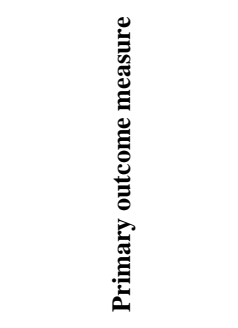 & 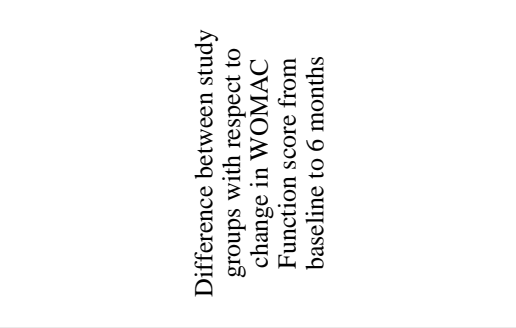 & 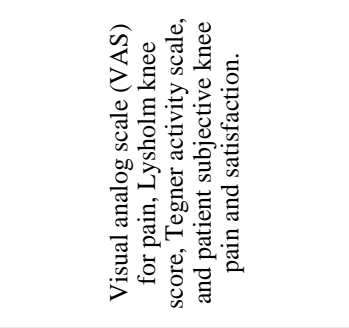 & 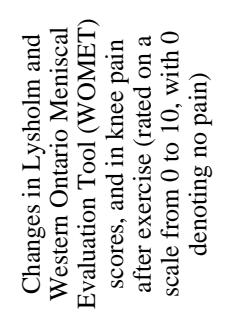 \\
\hline 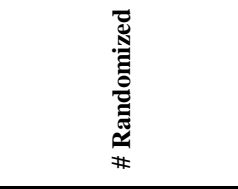 & $\bar{n}$ & $\stackrel{\varrho}{\varrho}$ & I \\
\hline 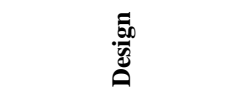 & $\underset{\varkappa}{E}$ & $\underset{\varkappa}{E}$ & $\underset{\sim}{E}$ \\
\hline$\stackrel{\varrho}{\Xi}$ & 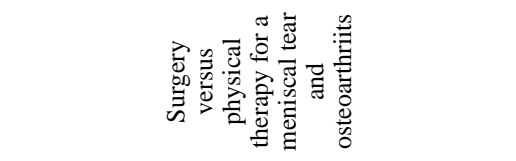 & 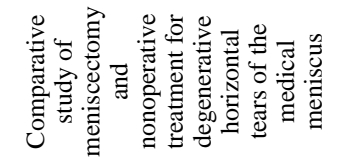 & 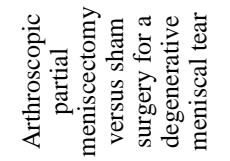 \\
\hline 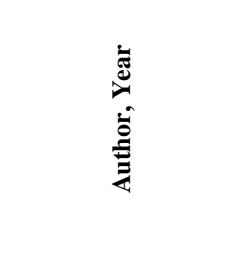 & 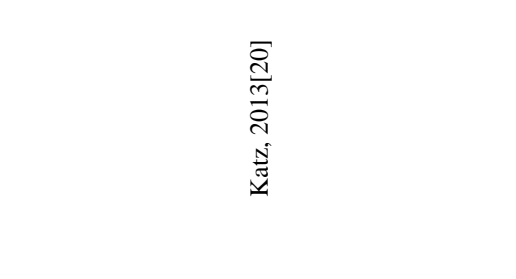 & 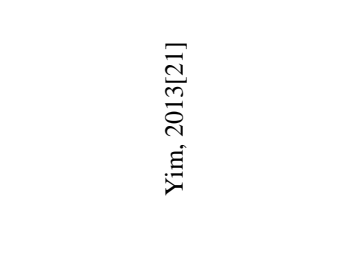 & 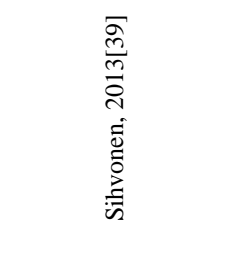 \\
\hline
\end{tabular}

Page 18 
Table 2

Statements in treatment guidelines relating to use of arthroscopic surgery for osteoarthritis

\begin{tabular}{|c|c|l|}
\hline Organization & Year & Arthroscopic Surgery Guideline \\
\hline AAOS[34] & 2013 & $\begin{array}{l}\text { We cannot recommend performing arthroscopy with lavage and/or debridement in patients with a primary diagnosis } \\
\text { of symptomatic osteoarthritis of the knee. Strength of Recommendation Strong }\end{array}$ \\
\hline ACR[35] & 2012 & Surgery not considered in most recent (2012) guidelines. \\
\hline OARSI[32, 33] & 2010 & $\begin{array}{l}\text { The role of joint lavage and arthroscopic debridement in knee OA are controversial. Although some studies have } \\
\text { demonstrated short-term symptom relief, others suggest that improvement in symptoms could be attributable to a } \\
\text { placebo effect. }\end{array}$ \\
\hline NICE[31] & 2008 & $\begin{array}{l}\text { Referral for arthroscopic lavage and debridement should not be offered as part of treatment for osteoarthritis, unless } \\
\text { the person has knee osteoarthritis with a clear history of mechanical locking (not gelling, 'giving way' or X-ray } \\
\text { evidence of loose bodies) }\end{array}$ \\
\hline
\end{tabular}

